\title{
Prognosis of liver abscess with no identified organism
}

\author{
Jai Hoon Yoon', Youn Jeong Kim ${ }^{2,3^{*}}$ and Sang $\| \mathrm{Kim}^{2,4}$
}

\begin{abstract}
Background: There are limited studies focusing on liver abscess with negative microbiological cultures. This study evaluated the clinical and prognostic differences of patients with culture-negative liver abscess (CNLA) compared to those with a positive culture (CPLA) and compared these factors between $K$. pneumoniae liver abscess (KLA) and E. coli liver abscess (ELA).

Methods: A retrospective study of the patients who admitted with a liver abscess at two tertiary hospitals in Korea from 2012 to 2016 was performed.

Results: Among a total of 402 patients with liver abscess, $61.2 \%$ had positive cultures. K. pneumoniae $(n=133)$ was the most common cause, followed by $E$. coli $(n=74)$. Patients with CPLA were significantly older $(p=0.02)$ and more frequently had cholelithiasis or biliary tract disease $(p=0.001)$ compared to patients with CNLA. In-hospital mortality $(p=0.63)$ and recurrence $(p=0.77)$ were no different between the two groups. The length of hospital stay was significantly longer in patients with CPLA $(p=0.03)$ compared with those with CNLA. Subgroup analysis for patients who received 3rd generation cephalosporins empirically showed that in-hospital mortality $(p=0.18)$ and recurrence $(p=0.27)$ were not also significantly different. Cholelithiasis, or biliary tract disease $(p=0.001)$, liver disease $(p=0.001)$, malignancy $(p=0.0001)$, and ESBL production $(p=0.0001)$ were found more frequently in patients with ELA compared with those with KLA.
\end{abstract}

Conclusions: The prognosis of the CNLA patients was similar to that of the CPLA patients, although the length of hospital stay was shorter in the CNLA patients. The epidemiologic and clinical characteristics of the ELA patients are somewhat different than those of the KLA patients.

Keywords: Liver abscess, Culture-negative, E.coli, K.pneumoniae

\section{Background}

Pyogenic liver abscess is a common intrabdominal infection, and population-based studies in North America, the United States, and Taiwan have shown that the incidence of pyogenic liver abscess ranges from 2.3 to 17.59 per 100,000 person-years [1-3]. E. coli is known to be a predominant liver abscess pathogen; however, over the past three decades, the leading pathogen has begun shifting to K. pneumoniae worldwide [4-8]. Despite advances in microbiologic techniques, a causative pathogen

\footnotetext{
* Correspondence: muze1004@catholic.ac.kr

${ }^{2}$ Department of Internal Medicine, College of Medicine, The Catholic University of Korea, Seoul, South Korea

${ }^{3}$ Division of Infectious Disease, Department of Internal Medicine, Incheon St. Mary's Hospital, College of Medicine, The Catholic University of Korea, \#56, Donsu-Ro, Bupyung-Gu, Incheon, South Korea

Full list of author information is available at the end of the article
}

is not identified in some cases of liver abscess. In one study, the sensitivity of the abscess culture was $90 \%$ for gram-positive cocci and $52 \%$ for gram-negative bacilli. However, the sensitivity was only $30 \%$ for gram positivecocci and 39\% for gram-negative bacilli in blood culture [9]. The best treatment for liver abscess is source control by drainage and administration of antibiotics that cover gram-negative bacilli, gram-positive cocci, and anaerobic bacteria $[10,11]$. Empirically, 3rd-generation cephalosporins plus metronidazole or $\beta$-lactam/ $\beta$-lactamase inhibitor are recommended initially, and antibiotics should later to be changed based on culture and antimicrobial susceptibility data. There are limited studies about liver abscess without a positive microbiological culture, and there are no guidelines for clinical practice in culture-negative liver abscess patients (CNLA). It remains unclear what kind of

(c) The Author(s). 2019 Open Access This article is distributed under the terms of the Creative Commons Attribution 4.0 International License (http://creativecommons.org/licenses/by/4.0/), which permits unrestricted use, distribution, and 
empiric antibiotics work better for CNLA patients. It is not clear whether the prognosis such as metastatic infection or mortality in CNLA patients is similar to that in patients with proven pathogens. This study was conducted to evaluate the clinical and prognostic differences in patients with CNLA compared to those with a positive microbiological culture, and to compare these factors between $K$. pneumoniae liver abscess (KLA) and E. coli liver abscess (ELA).

\section{Methods}

\section{Study design}

This retrospective study was conducted at Seoul St. Mary's Hospital of Catholic University and the tertiary hospital of Hanyang University in Korea from January 2012 to December 2016.

\section{Patients and definitions}

All hospitalized patients $>18$ years old who were admitted to these two hospitals who were diagnosed with a liver abscess at discharge were selected from the medical record database. Liver abscesses were diagnosed based on the clinical presentation, laboratory findings, and imaging studies such as computed tomography, ultrasonography, or magnetic resonance imaging. Microbiologic data were collected from at least 1 set of blood cultures obtained prior to starting antibiotics or from pus obtained through an invasive procedure. CNLA was diagnosed if no organism grew on the initial blood culture obtained prior to antibiotics or from pus obtained through an invasive procedure. Polymicrobial infection was defined as the presence of $\geq 2$ pathogens cultured from blood or pus. Metastatic infection was defined if a patient with liver abscess developed extrahepatic manifestations such as endophthalmitis, central nervous system infections, lung abscesses, and skin or soft tissue infections. Invasive procedures included aspiration, percutaneous drainage, and surgical interventions.

\section{Data collection}

The following data were collected: age, sex, underlying disease, laboratory findings, microbiologic data, treatment strategy, complications, and clinical outcomes. Inhospital mortality was used as the main outcome for assessing mortality in patients with a liver abscess.

\section{Microbiologic data}

Both aerobic and anaerobic cultures were performed for the blood and pus samples. Species identification and antimicrobial susceptibility were tested using VITEK automated systems (bioMérieux Vitek, USA), and interpreted according to guidelines established by the Clinical and Laboratory Standards Institute according to the Clinical and Laboratory Standards Institute (CLSI) criteria. Phenotypic confirmation of ESBL detection was performed using the double-disk diffusion method in our clinical microbiology laboratories, as recommended by the CLSI.

\section{Ethical approval}

The Institutional Review Board (IRB) of the Seoul St. Mary's Hospital (KC18REDI0751) and Hanyang University (2018-08-017-001) approved this study. The IRB waived the requirement to obtain written informed consent from the patients.

\section{Statistical analysis}

The independent sample $t$-test or Kruskal-Wallis test was used to analyze continuous variables, and the Chi-square test or Fisher's exact test was used for categorical variables. Statistical analysis was performed using SPSS 13.0 (SPSS Inc., Chicago, IL, USA), and a $p$-value of $<0.05$ was considered statistically significant.

\section{Results}

Comparison of patients with liver abscess with or without positive microbiological culture

\section{Demographic characteristics}

Among a total of 402 patients with liver abscess, etiologic organisms were found in 61.2\% $(n=246)$. Blood cultures were performed in 239 patients, and $41.4 \%$ ( $n=$ 99) were positive. Of 183 patients who underwent invasive procedures, $91.2 \%(n=167)$ were found to harbor etiologic organisms through abscess culture, and among these patients, 113 had negative blood cultures. Of 246 culture-proven liver abscesses (CPLA), K. pneumoniae was the most common pathogen found $(n=133)$, followed by $E$. coli $(n=74)$ (Fig. 1$)$. Table 1 shows the demographic characteristics of the patients with CPLA and CNLA. Those with CPLA were significantly older (median [IQR] 66 [8] vs. 61 [12] years old, $p=0.02$ ) and more frequently had cholelithiasis or biliary tract disease $(n=35(14.2 \%)$ vs. $n=6(3.8 \%), p=0.001)$ compared to patients with CNLA. Sex $(p=0.06)$; diabetes mellitus $(p=0.12)$, hypertension $(p=0.39)$, malignancy $(p=0.10)$ and transplant $(p=0.64)$ as a comorbidity; presentation with sepsis $(p=0.84)$; and ICU admission $(p=0.88)$ were no different between the two groups.

\section{Laboratory findings}

Table 2 shows a comparison of the laboratory data between the two groups. There were no significant differences in the white blood cell count $(p=0.69)$, AST $(p=0.70)$, ALT $(p=$ $0.53)$, alkaline phosphatase $(p=0.26), \mathrm{r}-\mathrm{GTP}(p=0.10)$, total bilirubin $(p=0.26)$, or creatinine $(p=0.06)$. The platelet count was significantly lower (median 167 vs. 212, $p=$ 0.001), and the CRP (median [IQR] 16.9 [8.7] vs. 13.3 [8.9], $p=0.01$ ) and albumin (median[IQR] 3.1[0.5] vs. 3.0[0.4], 


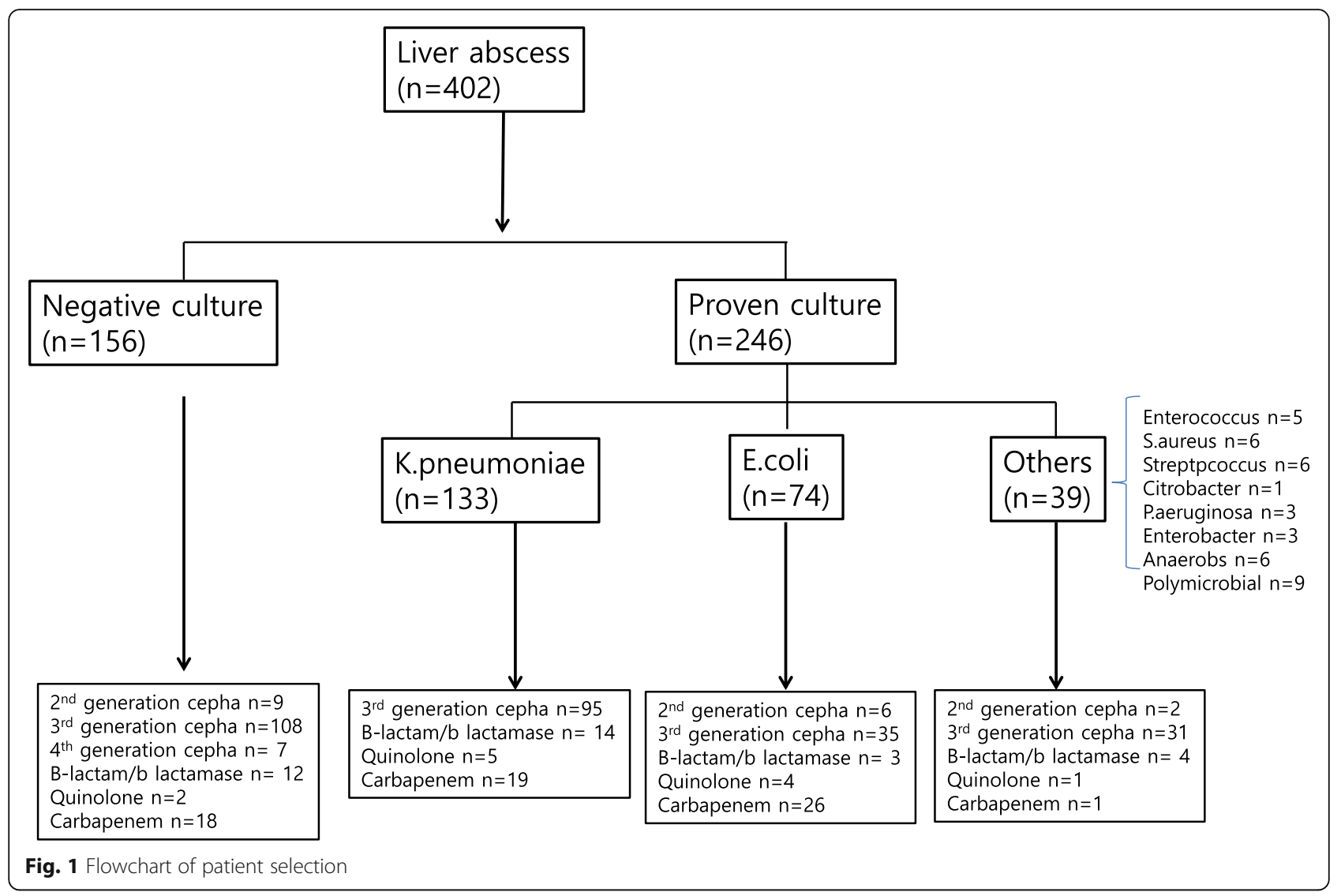

Table 1 Demographic characteristics of patients with liver abscess

\begin{tabular}{|c|c|c|c|c|c|c|c|}
\hline & \multirow{2}{*}{$\begin{array}{l}\text { CNLA } \\
(n=156)\end{array}$} & \multirow{2}{*}{$\begin{array}{l}\text { CPLA } \\
(n=246)\end{array}$} & \multicolumn{4}{|c|}{ Causative organisms } & \multirow[t]{2}{*}{$P$ value } \\
\hline & & & E.coli $(n=74)$ & $\begin{array}{l}\text { K.pneumoniae } \\
(n=133)\end{array}$ & $P$ value* & Other $(n=39)$ & \\
\hline Age, years, median (IQR) & $61(12)$ & $66(8)$ & $66.5(14.3)$ & 66 (18) & 0.95 & $66(7)$ & 0.02 \\
\hline Sex, male, n(\%) & $113(72.4 \%)$ & $156(63.4 \%)$ & 49 (66.2\%) & 79 (59.4\%) & 0.33 & $28(71.8 \%)$ & 0.06 \\
\hline \multicolumn{8}{|l|}{ Comorbidity } \\
\hline Diabetes mellitus & 35 (22.4\%) & $74(30.1 \%)$ & $24(33.8 \%)$ & $35(26.3 \%)$ & 0.26 & $14(35.9 \%)$ & 0.12 \\
\hline Hypertension & $63(40.4 \%)$ & 89 (36.2\%) & $26(35.1 \%)$ & 48 (36.1\%) & 0.89 & 15 (38.5\%) & 0.39 \\
\hline $\begin{array}{l}\text { Cholelithiasis and } \\
\text { biliary tract disease }\end{array}$ & $6(3.8 \%)$ & $35(14.2 \%)$ & $18(24.3 \%)$ & $11(8.3 \%)$ & 0.001 & $6(15.4 \%)$ & 0.001 \\
\hline Liver disease & $12(7.7 \%)$ & $33(13.4 \%)$ & $18(24.3 \%)$ & $10(7.5 \%)$ & 0.001 & $5(12.8 \%)$ & 0.07 \\
\hline Chronic kidney disease & $3(1.9 \%)$ & $5(2.0 \%)$ & $1(1.4 \%)$ & $3(2.3 \%)$ & 0.65 & $1(2.6 \%)$ & 0.94 \\
\hline Malignancy & $24(15.4 \%)$ & $54(22.0 \%)$ & 27 (36.5\%) & $11(8.3 \%)$ & 0.0001 & $16(41.0 \%)$ & 0.10 \\
\hline Transplant & $2(1.3 \%)$ & $2(0.8 \%)$ & $1(1.4 \%)$ & $1(0.8 \%)$ & 0.67 & $0(0 \%)$ & 0.64 \\
\hline Presentation as a sepsis & $5(3.2 \%)$ & $7(2.8 \%)$ & $1(1.4 \%)$ & $6(4.5 \%)$ & 0.23 & $0(0 \%)$ & 0.84 \\
\hline ICU admission & $22(14.1 \%)$ & 36 (14.6\%) & 11 (14.9\%) & $20(15.0 \%)$ & 0.97 & $5(12.8 \%)$ & 0.88 \\
\hline Bacteremia & - & 99 (40.2\%) & $21(28.4 \%)$ & 56 (42.1\%) & 0.05 & 22 (56.4\%) & - \\
\hline ESBL production & - & & 23 (31.1\%) & 3 (2.3\%) & 0.0001 & & \\
\hline
\end{tabular}

${ }^{*} P$ value was calculated on two groups of E.coli liver abscess and K.pneumoniae liver abscess 
Table $\mathbf{2}$ Laboratory findings of patients with liver abscess

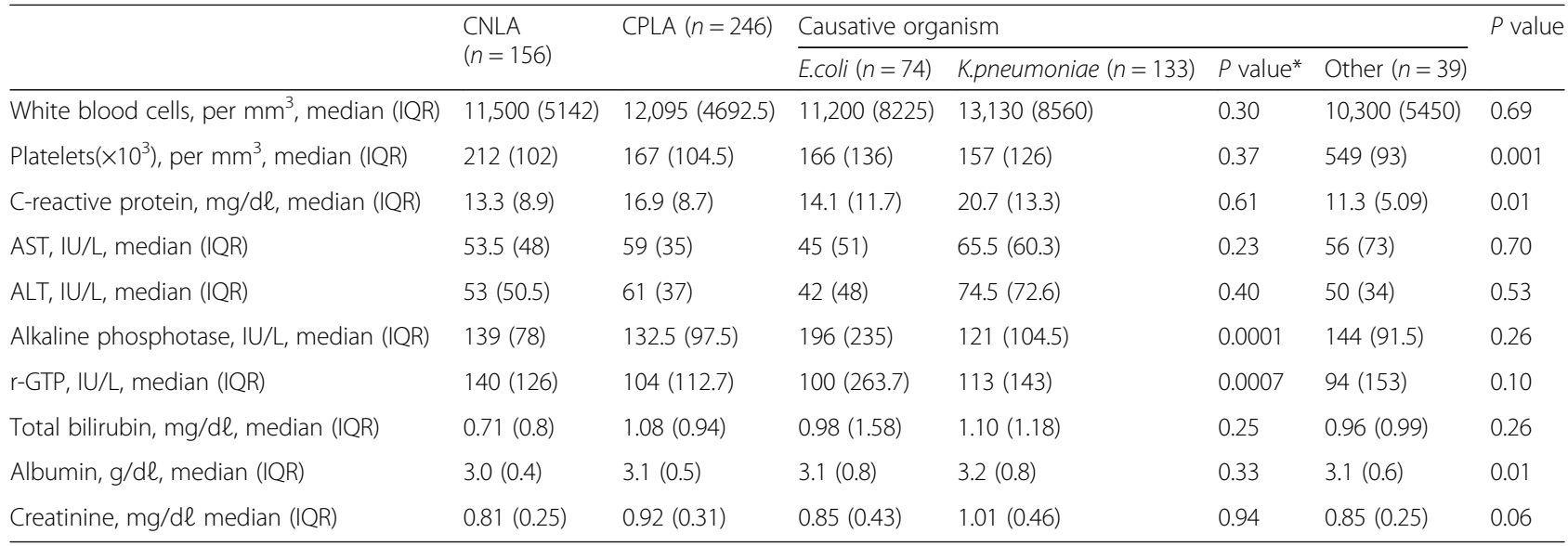

* $P$ value was calculated on two groups of E.coli liver abscess and K.pneumoniae liver abscess

$p=0.01)$ were higher in the CPLA patients compared with the CNLA patients.

\section{Treatment and clinical outcomes}

Invasive procedures, such as percutaneous drainage or surgery, were performed in $79.3 \%(n=195)$ of CPLA patients and $69.2 \%(n=108)$ of CNLA patients, and these differences were significant $(P=0.02)$ (Table 3). Metastatic infection developed more frequently in patients with CPLA $(n=12,4.9 \%)$ compared to those with CNLA $(n=2,1.3 \%)$; however, this difference was not significant $(p=0.05)$ (Table 3$)$. Empirically, the most commonly used antibiotics were 3rd generation cephalosporins in all groups, and these were more frequently prescribed in patients with CNLA $(n=108,69.2 \%)$ than in those with CPLA ( $n=161,65.4 \%)$ (Fig. 1). In-hospital mortality ( $p=$ $0.63)$ and recurrence $(p=0.77)$ were no different between the two groups. The length of hospital stay was significantly longer in CPLA patients (median [IQR] 17.9 [8] vs. $14.8[7.75], p=0.03$ ) compared with CNLA patients.

\section{Subgroup analysis for patients who received 3rd generation} cephalosporins empirically

In-hospital mortality (CPLA 5.0\% [8/161] vs. CNLA $1.9 \%[2 / 108], p=0.18$ ), recurrence (CPLA 5.0\% [8/161] vs. CNLA $8.3 \%$ [9/108], $p=0.27$ ) and metastatic infection (CPLA $5.0 \%[8 / 161]$ vs. CNLA $1.9 \%[2 / 108], p=$ 0.18 ) were not different between the two groups. The length of hospital stay was not different (CPLA median[IQR] 16 [11] days vs. CNLA median[IQR] 14.5 [10] days, $p=0.11$ ).

\section{Comparison of liver abscess patients with Klebsiella and E.coli \\ Demographic characteristics}

Table 1 shows a comparison between the demographic characteristics of patients with KLA $(n=133)$ and ELA $(n=74)$. Age $(p=0.95)$, diabetes mellitus $(p=0.26)$, hypertension $(p=0.89)$, and chronic kidney disease $(p=$ $0.65)$ as underlying diseases were not significantly different between the 2 groups. However, cholelithiasis, or biliary tract disease, was identified more often in patients with ELA ( $n=18,24.3 \%)$ compared to those with KLA $(n=11,8.3 \%) \quad(p=0.001)$. Liver disease (ELA $n=18$ $(24.3 \%)$ vs. KLA $n=10$ (7.5\%), $p=0.001)$ and malignancy (ELA $n=27(36.5 \%)$ vs. KLA $\mathrm{n}=11(8.3 \%), p=0.0001)$ were also found most frequently in patients with ELA. Patients with KLA had more bacteremia, although this difference was not significant (ELA $n=21(31.1 \%)$ vs. KLA $n=56(42.1 \%), p=0.05)$. ESBL-producing organisms were

Table 3 Treatment and clinical outcomes

\begin{tabular}{|c|c|c|c|c|c|c|c|}
\hline & \multirow{2}{*}{$\begin{array}{l}\text { CNLA } \\
(n=156)\end{array}$} & \multirow{2}{*}{$\begin{array}{l}\text { CPLA } \\
(n=246)\end{array}$} & \multicolumn{4}{|c|}{ Causative organism } & \multirow[t]{2}{*}{$P$ value } \\
\hline & & & E.coli $(n=74)$ & K.pneumoniae $(n=133)$ & $P$ value* & Other $(n=39)$ & \\
\hline Invasive procedure, n(\%) & $108(69.2 \%)$ & $195(79.3 \%)$ & $54(73.0 \%)$ & $108(81.2 \%)$ & 0.17 & $33(84.6 \%)$ & 0.02 \\
\hline Metastatic infection, n(\%) & $2(1.3 \%)$ & $12(4.9 \%)$ & $4(5.4 \%)$ & $7(5.3 \%)$ & 0.96 & $1(2.6 \%)$ & 0.05 \\
\hline In-hospital mortality, n(\%) & $6(3.8 \%)$ & $12(4.9 \%)$ & $5(6.8 \%)$ & $4(3.0 \%)$ & 0.20 & $3(7.7 \%)$ & 0.63 \\
\hline $\begin{array}{l}\text { Length of hospital stay, days, } \\
\text { median (IQR) }\end{array}$ & $14.8(7.75)$ & $17.9(8)$ & $20(15)$ & $18(9.5)$ & 0.09 & $17(16)$ & 0.03 \\
\hline Recurrence, n (\%) & $12(7.7 \%)$ & $17(6.9 \%)$ & $6(8.1 \%)$ & $5(3.8 \%)$ & 0.18 & $6(15.4 \%)$ & 0.77 \\
\hline
\end{tabular}

*P value was calculated on two groups of E.coli liver abscess and K.pneumoniae liver abscess 
observed more frequently in patients with ELA compared with those with KLA (ELA $n=23(31.1 \%)$ vs KLA $n=3$ (2.3\%), $p=0.0001)$.

\section{Laboratory findings}

There were no significant differences in the white blood cell count $(p=0.30)$, platelet count $(p=0.37)$, C-reactive protein $(p=0.61)$, AST $(p=0.23)$ or ALT $(p=0.40)$. Alkaline phosphatase $(p=0.26)$ was significantly higher (median [IQR] 196 [235] vs. 121 [104.5], $p=0.0001$ ), and the r-GTP (median [IQR] 100 [263.7] vs. 113 [143]. $p=$ 0.0007) was lower in the ELA patients compared with the KLA patients (Table 2).

\section{Treatment and clinical outcomes}

In both the ELA and KLA patients, 3rd generation cephalosporins were the most frequently used empiric antibiotics, followed by carbapenems (Table 3). Invasive procedures were performed more often in patients with KLA $(n=108,81.2 \%)$ than in those with ELA $(n=54$, $73.0 \%)$; however, this difference was not significant ( $p=$ $0.17)$. There was also no difference between the 2 groups in metastatic infections $(p=0.96)$, in-hospital mortality $(p=0.20)$ or recurrence $(p=0.18)$. The length of hospital stay was longer in ELA patients, although this was not significantly different (median [IQ] 20 [13] vs. 18 [9.5] days, $p=0.09$ ).

\section{Discussion}

The epidemiologic profile of liver abscess differs between countries. E. coli was a prevalent cause of liver abscess until the mid-1980s; however, since then, K. pneumoniae has become increasingly important as a liver abscess pathogen $[5,12-16]$. In our previous study, Klebsiella pneumoniae was also emerging as the main cause of liver abscess in our countries, followed by E. coli [17]. There have been reports of Klebsiella species associated with invasive syndromes showing extrahepatic complications in southeast Asian countries. However, there are few data on the clinical characteristics and prognosis for liver abscess with no etiologic organism identified or due to other pathogens.

There was no difference in the in-hospital mortality, metastatic infection or recurrence between patients with CNLA and CPLA. For patients with a liver abscess, empiric antibiotic coverage for gram-negative bacilli, grampositive cocci, and anaerobes is recommended, and 3rd generation cephalosporins and/or metronidazole or $\beta$ lactam/ $\beta$-lactamase inhibitor are the mainstay of treatment for liver abscess [10,18-20]. After pathogen identification, antibiotics should be modified based on microbiologic data and in vitro susceptibility. In clinical practice, physicians have some difficulty selecting antibiotics, especially for liver abscess due to unknown organisms. Clinicians may feel tempted to use broader, newer, and more expensive antibiotics. In our study, 3rd generation cephalosporins were the most frequently administered antibiotics in all patients with liver abscess. Subgroup analysis including patients with liver abscess who received 3rd generation cephalosporins showed that there was no difference in the in-hospital mortality and recurrence in CNLA and CPLA patients. It is acceptable that antibiotic selection for CNLA was similar to that for KLA because most patients with KLA, especially in cases of metastatic infection, had community-acquired infections, and the percentage of antibiotic resistant cases of Klebsiella pneumoniae was extremely low [15, 21]. However, host factors and local epidemiologic data must also be considered for antibiotic selection.

Our study demonstrated culture positivity among liver abscess patients in $41.4 \%$ by blood culture and $91.2 \%$ by invasive procedure. A population-based study in the United States found that $53.4 \%$ of patients had an identifiable organism on blood culture [1]. This finding suggests that advances in diagnostic methods have enhanced organism identification, and applications in real clinical situations are needed.

In our study, there were some differences in demographic characteristics between the ELA and KLA patients. ELA was associated with liver disease, biliary tract disease or malignancy, similar to previous results [13, 22, 23]. The percentage with ESBL production was higher in ELA than in KLA. In patients with ELA, underlying disease is is more likely to be associated with exposure to antibiotics or hospital admission, which may be associated with antibiotic resistance. Clinicians should consider broader spectrum antibiotics for patients with liver abscess if the patients have a malignancy or underlying biliary disease, as this may be suggestive of hospital acquired infection.

In our study, metastatic infection was also seen in patients with ELA, although there are several studies showing that the extrahepatic manifestation of liver abscess is typically a devastating complication in hypervirulent $K$. pneumoniae [12, 14, 17]. Further studies on virulence factors associated with invasive syndromes in E. coli are also needed.

Pyogenic liver abscess is a potentially life-threatening infection with high mortality. However, our study showed a relatively low in-hospital mortality of $5.7 \%$ compared to previous studies. [4, 13, 24, 25] In several studies, the mortality was associated with age, comorbidities, and prompt surgical modality including radiologic intervention $[1,26,27]$. We did not evaluate any factors associated with mortality in this study. However, the high percentage of invasive procedures performed in $75 \%$ of patients with liver abscess may be associated with favorable outcomes.

Our study has some limitations. It is a retrospective study that included only 2 hospitals. We did not 
examine factors related to organism identification; for example, the administration of antibiotics prior to culture collection might be associated with a false negative culture result.

\section{Conclusion}

Our study suggests that the prognosis including mortality, recurrence and metastatic infection was no different between the patients with CNLA and CPLA, although the length of hospital stay was shorter for CNLA patients compared with CPLA patients. The epidemiologic and clinical characteristics of the ELA patients are different from those of the KLA patients.

\section{Abbreviations}

CNLA: Culture-negative liver abscess; CPLA: Culture-positive liver abscess; ELA: E.coli liver abscess; KLA: K.pneumoniae liver abscess

\section{Acknowledgements}

None

\section{Authors' contributions}

JHY conceptualized and designed the paper, collected the data, and analyzed, wrote and drafted the paper. YJK collected and analyzed the data and critically reviewed the paper. SIK also critically reviewed the paper. All the authors read and approved the final paper.

\section{Funding}

This work was supported by the research fund of Hanyang University (HY- 2018). The funders did not have a role in the design or conduct of the study but did provide financial support for statistical consultation and English editing.

\section{Availability of data and materials}

The data analyzed during this study are included in this paper. Some of the datasets are available from the corresponding author upon reasonable request.

\section{Ethics approval and consent to participate}

The Institutional Review Board (IRB) of Seoul St. Mary's Hospital (KC18REDI0751) and Hanyang University (2018-08-017-001) approved this study. The study was a secondary data analysis and did not involve contact with individual patients so consent to participate was not applicable. Access to case data was approved by the Hanynag University and Seoul St. Mary's hospital.

\section{Consent for publication}

Not applicable.

\section{Competing interests}

The authors declare that they have no competing interest.

\section{Author details}

${ }^{1}$ Division of Gastroenterology, Department of Internal Medicine, Hanyang University College of Medicine, Seoul, South Korea. ${ }^{2}$ Department of Internal Medicine, College of Medicine, The Catholic University of Korea, Seoul, South Korea. ${ }^{3}$ Division of Infectious Disease, Department of Internal Medicine, Incheon St. Mary's Hospital, College of Medicine, The Catholic University of Korea, \#56, Donsu-Ro, Bupyung-Gu, Incheon, South Korea. ${ }^{4}$ Division of Infectious Disease, Department of Internal Medicine, Seoul St. Mary's Hospital, College of Medicine, The Catholic University of Korea, Seoul, South Korea.

Received: 8 January 2019 Accepted: 24 May 2019

Published online: 31 May 2019

\section{References}

1. Meddings L, Myers RP, Hubbard J, Shaheen AA, Laupland KB, Dixon E, Coffin C, Kaplan GG. A population-based study of pyogenic liver abscesses in the
United States: incidence, mortality, and temporal trends. Am J Gastroenterol. 2010;105(1):117-24.

2. Tsai FC, Huang YT, Chang LY, Wang JT. Pyogenic liver abscess as endemic disease, Taiwan. Emerg Infect Dis. 2008;14(10):1592-600.

3. Kaplan GG, Gregson DB, Laupland KB. Population-based study of the epidemiology of and the risk factors for pyogenic liver abscess. Clin Gastroenterol Hepatol. 2004;2(11):1032-8.

4. Seeto RK, Rockey DC. Pyogenic liver abscess. Changes in etiology, management, and outcome. Medicine (Baltimore). 1996;75(2):99-113.

5. Lederman ER, Crum NE. Pyogenic liver abscess with a focus on Klebsiella pneumoniae as a primary pathogen: an emerging disease with unique clinical characteristics. Am J Gastroenterol. 2005;100(2):322-31.

6. Lederman ER, Crum NF. Klebsiella liver abscess: a coast-to-coast phenomenon. Clin Infect Dis. 2005;41(2):273.

7. Corredoira Sanchez JC, Casariego Vales E, Ibanez Alonso MD, Rigueiro Veloso MT, Coira Nieto A, Alonso Garcia P, Rodriguez Feijoo A, Lopez Alvarez MJ, Varela Otero J. Pyogenic liver abscess: changes in etiology, diagnosis and treatment over 18 years. Rev Clin Esp. 1999;199(11):705-10.

8. Nah BK, Kim YS, Moon HS, Park KO, Kim SM, Lee YS, Yang HW, Seo SW, Kim $\mathrm{SH}$, Lee BS, et al. Recent changes of organism and treatment in pyogenic liver abscess. Taehan Kan Hakhoe Chi. 2003;9(4):275-83.

9. Chemaly RF, Hall GS, Keys TF, Procop GW. Microbiology of liver abscesses and the predictive value of abscess gram stain and associated blood cultures. Diagn Microbiol Infect Dis. 2003;46(4):245-8.

10. Hope WW, Vrochides DV, Newcomb WL, Mayo-Smith WW, lannitti DA. Optimal treatment of hepatic abscess. Am Surg. 2008;74(2):178-82.

11. Mavilia MG, Molina M, Wu GY. The evolving nature of hepatic abscess: a review. J Clin Transl Hepatol. 2016;4(2):158-68.

12. Fung CP, Chang FY, Lee SC, Hu BS, Kuo Bl, Liu CY, Ho M, Siu LK. A global emerging disease of Klebsiella pneumoniae liver abscess: is serotype K1 an important factor for complicated endophthalmitis? Gut. 2002;50(3):420-4.

13. Chen SC, Yen CH, Lai KC, Tsao SM, Cheng KS, Chen CC, Lee MC, Chou MC. Pyogenic liver abscesses with Escherichia coli: etiology, clinical course, outcome, and prognostic factors. Wien Klin Wochenschr. 2005;117(23-24): 809-15.

14. Gupta A, Bhatti S, Leytin A, Epelbaum O. Novel complication of an emerging disease: invasive Klebsiella pneumoniae liver abscess syndrome as a cause of acute respiratory distress syndrome. Clin Pract. 2018;8(1):1021

15. Siu LK, Yeh KM, Lin JC, Fung CP, Chang FY. Klebsiella pneumoniae liver abscess: a new invasive syndrome. Lancet Infect Dis. 2012;12(11):881-7.

16. Luo M, Yang XX, Tan B, Zhou XP, Xia HM, Xue J, Xu X, Qing Y, Li CR, Qiu JF, et al. Distribution of common pathogens in patients with pyogenic liver abscess in China: a meta-analysis. Eur J Clin Microbiol Infect Dis. 2016;35(10): 1557-65.

17. Yoon JH, Kim YJ, Jun YH, Kim SI, Kang JY, Suk KT, Kim DJ. Liver abscess due to Klebsiella pneumoniae: risk factors for metastatic infection. Scand J Infect Dis. 2014;46(1):21-6.

18. Lardiere-Deguelte $S$, Ragot E, Amroun K, Piardi T, Dokmak S, Bruno O, Appere F, Sibert A, Hoeffel C, Sommacale D, et al. Hepatic abscess: diagnosis and management. J Visc Surg. 2015;152(4):231-43.

19. Solomkin JS, Mazuski JE, Bradley JS, Rodvold KA, Goldstein EJ, Baron EJ, O'Neill PJ, Chow AW, Dellinger EP, Eachempati SR, et al. Diagnosis and management of complicated intra-abdominal infection in adults and children: guidelines by the surgical infection society and the Infectious Diseases Society of America. Clin Infect Dis. 2010;50(2):133-64.

20. Zerem E, Omerovic M, Zerem O. What is the optimal treatment for hepatic abscess and infected biloma? Gastrointest Endosc. 2015;82(5):971-2.

21. Pomakova DK, Hsiao CB, Beanan JM, Olson R, MacDonald U, Keynan Y, Russo TA. Clinical and phenotypic differences between classic and hypervirulent Klebsiella pneumonia: an emerging and under-recognized pathogenic variant. Eur J Clin Microbiol. 2012;31(6):981-9.

22. Chen SC, Wu WY, Yeh CH, Lai KC, Cheng KS, Jeng LB, Wang PH, Lin DB, Chen CC, Lee MC, et al. Comparison of Escherichia coli and klebsiella pneumoniae liver abscesses. Am J Med Sci. 2007;334(2):97-105.

23. Shelat VG, Chia CLK, Yeo CSW, Qiao W, Woon W, Junnarkar SP. Pyogenic liver abscess: does Escherichia Coli cause more adverse outcomes than Klebsiella Pneumoniae? World J Surg. 2015;39(10):2535-42.

24. Wang YC, Yang KW, Lee TYP, Lin CL, Liaw GW, Hung DZ, Kao CH, Chen WK, Yang TY. Increased risk of pyogenic liver abscess in patients with alcohol intoxication: a population-based retrospective cohort study. Alcohol. 2017; $64: 23-8$. 
25. Chu KM, Fan ST, Lai EC, Lo CM, Wong J. Pyogenic liver abscess. An audit of experience over the past decade. Arch Surg. 1996;131(2):148-52.

26. Sohn SH, Kim KH, Park JH, Kim TN. Predictors of mortality in Korean patients with pyogenic liver abscess: a single center, retrospective study. Korean J Gastroenterol. 2016;67(5):238-44.

27. Chen SC, Tsai SJ, Chen CH, Huang CC, Lin DB, Wang PH, Chen CC, Lee MC. Predictors of mortality in patients with pyogenic liver abscess. Neth J Med. 2008;66(5):196-203.

\section{Publisher's Note}

Springer Nature remains neutral with regard to jurisdictional claims in published maps and institutional affiliations.

Ready to submit your research? Choose BMC and benefit from:

- fast, convenient online submission

- thorough peer review by experienced researchers in your field

- rapid publication on acceptance

- support for research data, including large and complex data types

- gold Open Access which fosters wider collaboration and increased citations

- maximum visibility for your research: over $100 \mathrm{M}$ website views per year

At $\mathrm{BMC}$, research is always in progress.

Learn more biomedcentral.com/submissions 